\title{
Sensor-Based Technology: Bringing Value to People with Diabetes and the Healthcare System in an Evolving World
}

\author{
Judith L Glennie', Lori Berard², Fleur Levrat-Guillen ${ }^{3}$ \\ IJ.L. Glennie Consulting Inc., Aurora, Ontario, Canada; ${ }^{2}$ Nurse Consultant, Pink Pearls Inc, Winnipeg, Manitoba, Canada; ${ }^{3}$ Abbott Diabetes Care, \\ Maidenhead, UK \\ Correspondence: Fleur Levrat-Guillen, Abbott Laboratories Ltd, Abbott House, Vanwall Business Park, Maidenhead, Berkshire, SL6 4XE, UK, \\ Tel +44 7584I08032, Email fleur.levrat@abbott.com
}

\begin{abstract}
Purpose: Evidence demonstrates that glucose-sensing technologies have enabled effective glycemic control for adults and children with type 1 diabetes (T1DM) or adults with type 2 diabetes (T2DM) on insulin therapy or non-insulin therapy. Here, we report on the wider value of glucose-sensing technology from the perspectives of person living with diabetes (PWD), healthcare providers (HCPs), and healthcare policy stakeholders.

Methodology: Literature searches were conducted to identify published records and analysis, including across various healthcare organizations and agencies, of the impact of the FreeStyle Libre ${ }^{\circledR}$ flash glucose monitoring system in diabetes. These findings were combined with the outcomes of three healthcare attitudes surveys among PWD and diabetes healthcare professionals in Canada, including two commissioned for this purpose.

Results: Clinical trials data and real-world evidence have proven the benefits of the FreeStyle Libre system on limiting hypoglycemia, lowering HbAlc, optimizing metrics of glucose control and reducing hospital admissions. These benefits are accompanied by improvements in patients' quality of life, work productivity, and savings to the health system. The FreeStyle Libre system has created an opportunity to change the organization and delivery of care, including during COVID-19 restrictions on access to standard care, thus generating system-wide benefits in addition to those accrued by patients and HCPs.

Conclusion: Evidence-based improvements in glucose control for PWD using flash glucose monitoring are accompanied by increased treatment satisfaction and quality of life. Telemedicine with such remote monitoring systems increases the opportunities for simultaneous review of glucose data with HCPs and shared decision-making, thus encouraging adherence with treatment.
\end{abstract}

Keywords: glucose monitoring, telemedicine, healthcare costs, organization of care, treatment adherence

\section{Background and Introduction}

Central to good diabetes care for people living with diabetes (PWD) is the self-monitoring of blood glucose (SMBG). ${ }^{1-3}$ It is critical in the detection and/or prevention of hypoglycemia and provides PWD with an important tool for assessing blood-glucose levels before and after meals, exercise, and a range of daily activities. However, SMBG only provides an isolated glucose reading at the moment of measurement with no additional context, and its accuracy may vary depending on the technique of the user. ${ }^{4}$ More importantly, SMBG testing can be painful, which has consequences for both the frequency of testing and the quality of life (QOL) for the person with diabetes. ${ }^{5}$ Poor adherence with SMBG testing has consequences for long-term diabetes health, since reduced frequency of testing is associated with increased $\mathrm{HbAlc}$ levels ${ }^{6}$ such that the majority of people with type 1 diabetes (T1DM) or type 2 diabetes (T2DM) fail to meet HbA1c targets, both in the $\mathrm{US}^{7}$ and in Canada, ${ }^{8}$ putting them at increased risk of diabetes-related complications.

Continuous glucose monitoring (CGM) technologies address the acknowledged limitations of SMBG testing. CGM sensors measure glucose in the subcutaneous interstitial fluid using a thin filament inserted under the skin. This largely eliminates the need for SMBG tests and test strips, except at times when sensor-glucose readings do not match the 
symptoms experienced by the user. The FreeStyle Libre ${ }^{\circledR}$ flash glucose monitoring system takes a glucose reading every minute, which is provided to the user each time they scan their sensor with a smartphone app or a system reader. It also provides the user with a glucose trend arrow that allows the user to see whether their glucose is falling or rising and how fast. Thus, PWD using the FreeStyle Libre system have access to their glucose data in real-time directly from their device and are provided with important information on their glucose levels that is actionable in real time. Here, we review the objective evidence that supports the use of the FreeStyle Libre system as part of effective glycemic control and diabetes management for PWD. We examine the global outcomes related to using the FreeStyle Libre system in glucose control; however, the focus of our analysis is the potential benefits of providing access to the FreeStyle Libre system for the care of children and adults with diabetes in Canada.

\section{Methodology}

\section{Literature Search Details}

The search strategies were developed and tested through an iterative process by an experienced medical information specialist in consultation with the review author. Using the OVID platform, we searched Ovid MEDLINE ${ }^{\circledR}$ ALL, Embase, and APA PsycINFO. We also searched CINAHL on the EBSCO platform and Web of Science. These databases provide comprehensive coverage of journals, abstracts, and other published content across the biomedical, clinical, pharmacological, psychological, nursing, and allied health sciences. Because the coverage of Medline and Embase overlaps comprehensively with the PubMed ${ }^{\circledR}$ and SCOPUS ${ }^{\circledR}$, these databases were not interrogated.

Strategies utilized a combination of controlled vocabulary (eg, "Diabetes Mellitus", "Blood Glucose SelfMonitoring", "Biosensing Techniques/is, mt [Instrumentation, Methods]") and keywords (eg, "T2 DM", "FGMS", "FreeStyle"). Vocabulary and syntax were adjusted across the databases. When possible, animal-only records were removed from the results. Results were limited to the English language and the publication years 2015 to the present. In addition to the formal literature search, the internet and grey literature sources outside of commercial publishing organizations were reviewed to glean insights on relevant topics.

\section{Survey Methodologies Diabetes Canada Survey}

A survey was conducted by Diabetes Canada to understand the pandemic's impact on people affected by diabetes and their health, how they have managed and adapted, and their needs going forward. This survey was conducted using SurveyMonkey and available to the public between May 14 and June 17, 2021. It was publicized on Diabetes Canada's social media channels, through partners and via e-blast. In total, 780 people responded to the survey with results in the document rounded to the nearest decimal point.

\section{Ipsos Survey of PWD}

Ipsos is a market research and consulting firm, which conducted a survey on behalf of Abbott Diabetes Care during the COVID-19 pandemic, covering areas of diabetes management and support; the role of government in supporting access for PWD, and the shift to virtual care - in general and as a result of COVID-19. A sample of 1500 adult Canadians with diabetes was surveyed online via the Ipsos I-Say panel, with $90 \%$ of the sample having T2DM, while $10 \%$ of the sample had T1DM. Quotas and weighting were employed to ensure that the sample's composition reflects that of the Canadian population with diabetes according to census information.

\section{Banty Survey of HCPs}

Abbott Diabetes Care (Canada) conducted a survey of 44 HCPs in the Hamilton, Ontario region to gain an understanding of their experiences and perspectives in using virtual methods to manage patients living with diabetes. The intention was to determine the current state of virtual diabetes care and to assess how the Freestyle Libre system supports the continuity of care in PWDs, to enable HCPs in their efforts to provide care. Survey responses were aggregated and anonymized, with the intention of sharing with stakeholders such as Diabetes Canada as well as the Ontario Government. 


\section{Results}

\section{The Efficacy of the FreeStyle Libre System in Clinical Trials and Real-World Studies}

Numerous studies have proven the clinical benefits of flash glucose monitoring in people with T1DM or T2DM treated with different intensive insulin regimens, including multiple daily injections with insulin (MDI) and insulin pump therapy. ${ }^{9-13}$ In the IMPACT randomised clinical trial (RCT), over 6 months in well-controlled adults with T1DM, ${ }^{9}$ the FreeStyle Libre system was associated with a $38 \%$ reduction in time spent in hypoglycemia $<3.9 \mathrm{mmol} / \mathrm{L}(70 \mathrm{mg} / \mathrm{dL})$ compared to SMBG testing, including a 50\% reduction in nocturnal hypoglycemia, whilst maintaining HbA1c levels. The REPLACE RCT ${ }^{10}$ evaluated the FreeStyle Libre system in adults with T2DM on intensive insulin therapy. After 6 months, subjects using the FreeStyle Libre system reduced their time in hypoglycemia by 43\%, compared to the SMBG arm, including a $54 \%$ reduction in nocturnal hypoglycemia. Although HbAlc was not reduced compared to SMBG testing in the IMPACT or REPLACE RCTs, ${ }^{9,10}$ the single-arm SELFY study ${ }^{11}$ in children and young adults with T1DM showed that using the FreeStyle Libre system over 8 weeks was associated with a $0.4 \%$ reduction in HbA1c compared to standard of care with SMBG testing. An open-label 12-week prospective randomised controlled study by Yaron et al in people with $\mathrm{T} 2 \mathrm{DM}$ on insulin therapy ${ }^{12}$ demonstrated a significant reduction in HbAlc in the FreeStyle Libre system group $(-0.85 \%[ \pm 0.45])$ compared to an SMBG testing group $(-0.32 \%[ \pm 0.39])(\mathrm{p}<0.0001)$.

Subsequent to these prospective RCTs, a meta-analysis of 25 real-world observational studies confirmed that flash glucose monitoring is associated with reductions in HbAlc for children and adults with T1DM, and for adults with T2DM on insulin therapy. ${ }^{13}$ Mean change in HbA1c 2-4 months after starting the FreeStyle Libre system was $-0.56 \%$ for adults and $-0.54 \%$ in children and adolescents. Based on regression analysis, the degree of change in HbAlc correlated with the initial HbAlc of the study population, ie, the higher the starting HbA1c, the greater the reduction after starting with the FreeStyle Libre system. These observations have been further reinforced by the Association of British Clinical Diabetologists (ABCD) FreeStyle Libre audit ${ }^{14}$ that found a mean reduction in HbAlc of $-0.5 \% 6$ months after starting the system across a population of 3183 FreeStyle Libre system users. In this audit, use of flash glucose monitoring was also associated with reduced incidence of impaired awareness of hypoglycemia and less diabetes distress.

Looking specifically at T2DM, a retrospective chart review across 18 medical centres in 3 European countries ${ }^{15}$ evaluated the impact of changing to the FreeStyle Libre system in people with T2DM on basal bolus insulin therapy. After 3 to 6 months, HbA1c was reduced significantly, by $-0.9 \%$ in Germany and Austria, and by $-0.8 \%$ in France. A retrospective chart review across 6 diabetes centres in Canada of 91 adults with T2DM on basal-only insulin also showed a $-0.8 \%$ reduction in HbAlc 3-6 months after starting to use the FreeStyle Libre system. ${ }^{16}$ Recently, data have also shown that flash glucose monitoring is associated with reductions in HbA1c in people with T2DM on non-insulin therapies. A prospective study on 48 subjects with T2DM saw a $0.46 \%$ reduction in HbA1c after 24 weeks with the FreeStyle Libre system. ${ }^{17}$ A larger, retrospective observational review of 728 adults with T2DM indicated a $-1.6 \%$ fall in $\mathrm{HbA1c}$ for those on non-insulin therapy, between 2 and 10 months after starting the FreeStyle Libre system. ${ }^{18}$

\section{The Emergence of Glucometrics That Go Beyond HbAlc}

The advent of sensor-based glucose-monitoring technologies and their ability to report on glucose levels in real-time has enabled the objective assessment of both the rates and the risk of hypoglycemia in patients who are most vulnerable. This is important, since hypoglycemia is a major limiting factor in the glycemic management of people with T1DM or T2DM. ${ }^{19}$

Along with assessments of time spent with low glucose below $3.9 \mathrm{mmol} / \mathrm{L}(70 \mathrm{mg} / \mathrm{dL})$ or with clinically important hypoglycemia below $3.0 \mathrm{mmol} / \mathrm{L}(54 \mathrm{mg} / \mathrm{dL})$, the availability of CGM data has led to the development of new metrics that can be used to assess and improve glucose control. Thus, time in range (TIR) is gradually becoming a useful metric for people who use the FreeStyle Libre system and CGM devices in their daily diabetes care. ${ }^{20}$ TIR refers to the amount of time that a person with diabetes spends within a specified target glucose range, typically $3.9-10 \mathrm{mmol} / \mathrm{L}$ ( $70-180 \mathrm{mg} /$ $\mathrm{dL}$ ). Time below range (TBR) $<3.9 \mathrm{mmol} / \mathrm{L}(70 \mathrm{mg} / \mathrm{dL})$ and time above range (TAR) $>10 \mathrm{mmol} / \mathrm{L}(180 \mathrm{mg} / \mathrm{dL})$ are also important measures that quantify periods when glucose levels are below or above target. These are now accepted critical metrics when assessing the glycemic profile of a person with diabetes. The time spent in any of these ranges can be 
described either as the percentage of glucose values recorded each day or as the number of hours/minutes per day spent in that range, and consensus targets have been developed for use in daily diabetes care. ${ }^{21}$ The goal for effective and safe glucose control is to increase \%TIR while reducing \%TBR.

The value of TIR as a metric for optimizing glucose control is validated by studies showing the correlation between TIR and HbAlc levels ${ }^{22,23}$ and the association of TIR with microvascular and macrovascular complications of diabetes. An inverse relationship has been demonstrated between TIR and risk of diabetic retinopathy in T1DM and T2DM, ${ }^{24,25}$ with nephropathy in T1DM, ${ }^{24}$ and with neuropathy in $\mathrm{T} 2 \mathrm{DM}^{26}{ }^{26}$ Notably, increased TIR has been correlated with improvements in markers of cardiovascular disease (CVD) and with reductions in risk of all-cause and CVD mortality in T2DM. ${ }^{27,28}$

Other accepted metrics that have emerged from CGM data are glucose variability, expressed as the coefficient of variation (CV) of glucose, ${ }^{29}$ and the glucose management indicator (GMI), which is a measure of short-term glucose exposure that allows treatment adjustment to be fine-tuned when used in conjunction with a recent $\mathrm{HbAlc}$ test reading. ${ }^{30}$

\section{Visualizing Glucose Control Using CGM and Flash Glucose Monitoring Data}

Standard reports that are generated for the FreeStyle Libre and CGM systems also include the user's ambulatory glucose profile (AGP). The AGP displays large amounts of glucose data from across several days or weeks as if all the readings had occurred in a single 24-hour period. ${ }^{31}$ The visually impactful format allows HCPs and PWD to identify patterns and trends in daily glucose control, including those that may be of clinical importance; and pinpoints times within each day of increased risk of hypoglycemia or hyperglycemia. Significantly, the AGP helps visualize glycemic variability which is emerging as an important risk factor for diabetes complications, independent of average blood glucose levels. ${ }^{32}$ This is a powerful tool for PWD and their HCPs to examine options for improving diabetes control.

These new metrics supplement and overcome many of the limitations of HbAlc, which has long been established as the primary predictor for the risk of long-term diabetes complications. Because $\mathrm{HbA1c}$ only reflects average glucose levels over the previous 2-3 months, it does not provide information on glucose variability or the incidence of hypo- or hyperglycemia at any point in time during the day or night.

\section{Access to the FreeStyle Libre System}

The wider use of CGM and flash glucose monitoring technologies has prompted various professional and government agencies to assess their role in the care and management of PWD. A summary of these assessments as they pertain to adoption of the FreeStyle Libre system by the Canadian healthcare system is provided below. The current state of funding for the FreeStyle Libre system by Canadian jurisdictions (as of September 2021) is also summarized (Table 1).

\section{Canadian Agency for Drugs and Technologies in Health (CADTH)}

CADTH is an independent, not-for-profit organization responsible for providing Canada's healthcare decision-makers with evidence about the effective use of drugs and medical devices. At the time that the FreeStyle Libre system was approved by Health Canada ${ }^{33}$ in 2016, CADTH provided no overall recommendation, but it did highlight a number of potential benefits of the system for people with T1DM or T2DM. In September 2020, CADTH published two more reports: a comprehensive technology review ${ }^{34}$ as well as implementation advice ${ }^{35}$ regarding the FreeStyle Libre system. These reports were generated in response to a request from public drug plans, who were under increasing pressure from patients and HCPs to provide health-service funded access to this system.

The CADTH technology review synthesized the findings of health technology assessments (HTA) undertaken by Ontario (via OHTAC) and Quebec (via INESSS), to facilitate funding decisions by jurisdictions that had not done their own HTA reviews. Both HTAs recommended funding the FreeStyle Libre system with conditions and/or criteria. (See below for summaries of the OHTAC and INESSS recommendations and reimbursement in Ontario and Quebec, respectively.) The CADTH report highlighted the therapeutic benefits supported by evidence - namely, the reduced frequency and duration of hypoglycemia events in T1DM and T2DM for people using intensive insulin therapy; and, improvement in time spent in the target glucose range in T1DM. 
Table I Summary of Current Canadian Public Payer Reimbursement for the FreeStyle Libre System ${ }^{\mathrm{a}}$

\begin{tabular}{|c|c|}
\hline Jurisdiction & Criteria \\
\hline Ontario $^{86}$ & $\begin{array}{l}\text { - Patients managing diabetes with insulin } \\
\text { - Maximum } 33 \text { sensors per } 365 \text { days }\end{array}$ \\
\hline Quebec $^{87}$ & $\begin{array}{l}\text { People } 18+\text { who meet all of the following criteria: } \\
\text { - On intensive insulin therapy with at least } 3 \text { injections daily or using a pump; and, } \\
\text { - Having frequent hypoglycemia in the last year. } \\
\text { Initial authorization given for } 6 \text { months to evaluate patient's capacity to use FreeStyle Libre system and } \\
\text { wear the sensor. }\end{array}$ \\
\hline Yukon & $\begin{array}{l}\text { Announcement of a program to provide funding for continuous glucose monitoring to all Yukoners with } \\
\text { TIDM from age } 2 \text { to } 18 \text { (as of April I, 202I). }{ }^{88} \\
\text { - NOTE: specific devices not identified in the announcement. } \\
\text { A program is also being developed for Yukoners over } 18 \text { years of age with TIDM, to have access to fully } \\
\text { funded flash glucose monitors. }\end{array}$ \\
\hline Manitoba $^{90}$ & $\begin{array}{l}\text { On September 14, } 2021 \text {, the Manitoba government established a new program to pay for advanced } \\
\text { glucose monitors for individuals aged } 25 \text { years and younger with Type I diabetes. PWD who meet } \\
\text { eligibility criteria will have the option of either continuous or flash glucose monitors. }\end{array}$ \\
\hline Saskatchewan ${ }^{91,92}$ & $\begin{array}{l}\text { Full coverage for continuous and flash glucose monitors and related supplies for children and youth under } \\
18 \text { who are insulin dependent (as of June I, 202I). }\end{array}$ \\
\hline $\begin{array}{l}\text { Non-insured Health Benefits } \\
\text { (NIHB) Program }\end{array}$ & Reimbursement on a case-by-case basis ${ }^{\mathbf{b}}$ \\
\hline
\end{tabular}

Notes: ${ }^{a}$ Of note, most private drug plans in Canada provide coverage for the FreeStyle Libre system (Abbott information on file). ${ }^{b}$ Abbott Laboratories Inc. (Canada). Information on file.

For the second report, CADTH convened an implementation panel tasked with providing the context for the HTA and identifying additional factors that should be considered by policy makers as part of their decision process (eg, specific groups of patients who may benefit). The panel identified a number of subgroups within the insulin-treated population who would be predicted to benefit from using the FreeStyle Libre system. The panel noted additional clinically relevant benefits, including enhanced patient comfort, convenience, and independence. Improved patient compliance with glucose monitoring and better disease management through analysis of glycemic trends were also noted.

More recently, CADTH completed a review of the FreeStyle Libre systems in the pediatric T1DM population, ${ }^{36}$ concluding that the evidence indicates that the systems may improve QOL, patient satisfaction, diabetes distress, self-efficacy, and frequency of glucose monitoring compared to SMBG. The report noted that findings related to $\mathrm{HbA1c}$, glucose TIR metrics, and adverse events were mixed; but ultimately concluded that the use of either of the FreeStyle Libre systems is associated with improved clinical outcomes in pediatric populations with T1DM.

\section{Ontario Health Technology Advisory Committee (OHTAC)}

OHTAC makes evidence-based recommendations on which healthcare services and devices should be publicly funded, ${ }^{37}$ which require approval from Ontario Health management before they can be adopted. ${ }^{38}$

The OHTAC review ${ }^{39}$ recommended public funding of the FreeStyle Libre system for 2 groups of patients: 1) People with T1DM who experience recurrent hypoglycemia despite frequent SMBG testing and efforts to optimize insulin therapy and 2) People with T2DM requiring intensive insulin therapy who experience recurrent hypoglycemia despite frequent SMBG testing and efforts to optimize insulin therapy. The Ontario Drug Benefit Program has reimbursed the FreeStyle Libre system since September 16, 2019 (see reimbursement details in Table 1) in part based on this recommendation. 


\section{Institut National d'Excellence en Santé et Services Sociaux (INESSS)}

INESSS is the HTA body for the province of Quebec ${ }^{40}$ and evaluates new medications and makes recommendations to the Minister of Health and Social Services regarding funding on Quebec's List of Medications (ie, formulary for the basic drug insurance plan) and the List of Medications - Institution (ie, medications used in hospitals).

INESSS recommended in October $2018^{41}$ that the FreeStyle Libre system be added to the Quebec drug plan for selfmonitoring of glycemia in patients on insulin therapy (with some caveats related to the pricing of the system). Régie de l'assurance maladie du Québec (RAMQ) funded the FreeStyle Libre system from July 2019 and updated its coverage criteria in April 2020 subsequent to a second INESSS evaluation, ${ }^{42}$ with further updates being implemented in January 2021. This most recent update (see Table 1) recognized the value of the FreeStyle Libre system for patients on intensive insulin therapy who experience frequent severe hypoglycemia despite the adoption of a glycemic management plan. The initial RAMQ funding request is only for 6 months in order to evaluate the patient's ability to wear the sensor and use the system. Thereafter, funding requests are renewed for a period of 12 months.

\section{Diabetes Canada}

As a consequence of the rapid uptake of newer glucose monitoring technologies, Diabetes Canada updated their clinical practice guidelines for glucose monitoring in adults and children with diabetes in September 2021. ${ }^{43}$ Although periodic $\mathrm{HbAlc}$ testing is recommended, the 2021 guidance recognizes that HbAlc tests do not support immediate or short-term decisions on diabetes self-management. Flash glucose monitoring is now indicated in preference to SMBG for all individuals with T1DM using basal-bolus insulin therapy or insulin-pump therapy, specifically to increase TIR and reduce the incidence and duration of hypoglycemia, as well as increase treatment satisfaction. This emphasis on flash glucose monitoring is extended to adults with T2DM using basal-bolus insulin therapy who are not achieving HbA1c targets. Diabetes Canada also recognizes the need for clinicians to be more aware of the new glucometrics of TIR, TBR, TAR, and glycemic variability, as well as familiarity with the AGP report format.

Separate from this guidance, wider access to flash glucose monitoring in Canada has prompted several surveys to evaluate perspectives on the system among PWD and HCPs, particularly in the context of the COVID-19 pandemic. These surveys provide additional context for the value of the system.

\section{Canadian Surveys on the Use of Technology in Glucose Monitoring}

Three Canadian surveys of PWD and HCPs have examined the role of glucose monitoring technologies (particularly the FreeStyle Libre system) in general and in the context of the COVID-19 pandemic.

\section{Diabetes Canada Survey}

Diabetes Canada conducted a survey in May/June 2021 centered on the impact of the COVID-19 pandemic on individuals and caregivers affected by diabetes, focusing on their health, how they have managed and adapted, and their needs going forward. ${ }^{44} \mathrm{~A}$ total of 780 people responded, with $86 \%$ being people living with diabetes and $11 \%$ from the diabetes caregiver community and 3\% who were both. Respondents were evenly split between T1DM and T2DM, almost two-thirds were women, with more-than half of the respondents being aged 60 years or older.

As shown in Figure 1, COVID-19 has been stressful for PWD and their caregivers. Seventy-five percent expressed concerns about contracting the virus which was compounded by feelings of isolation or loneliness (41\%), anxiety (46\%), and concerns about their mental health (37\%).

Continuity of care has been impacted for two-thirds of respondents; and, about half have had virtual medical visits since the pandemic started. Of interest was that $73 \%$ of the respondents felt that virtual appointments were convenient, with $44 \%$ reporting a comparable experience to in-person care, with almost $40 \%$ indicating that they would prefer more virtual visits in the post-pandemic world (Figure 1). Leveraging technology has been a key part of this shift to virtual care. Forty-seven percent of respondents reported use of advanced glucose monitoring systems, and $72 \%$ indicated that they share their diabetes data digitally with their clinics. It is clear that technology is becoming an important tool in providing continuous diabetes support through virtual diabetes care, both during COVID-enforced restrictions on access to regular care and beyond. 


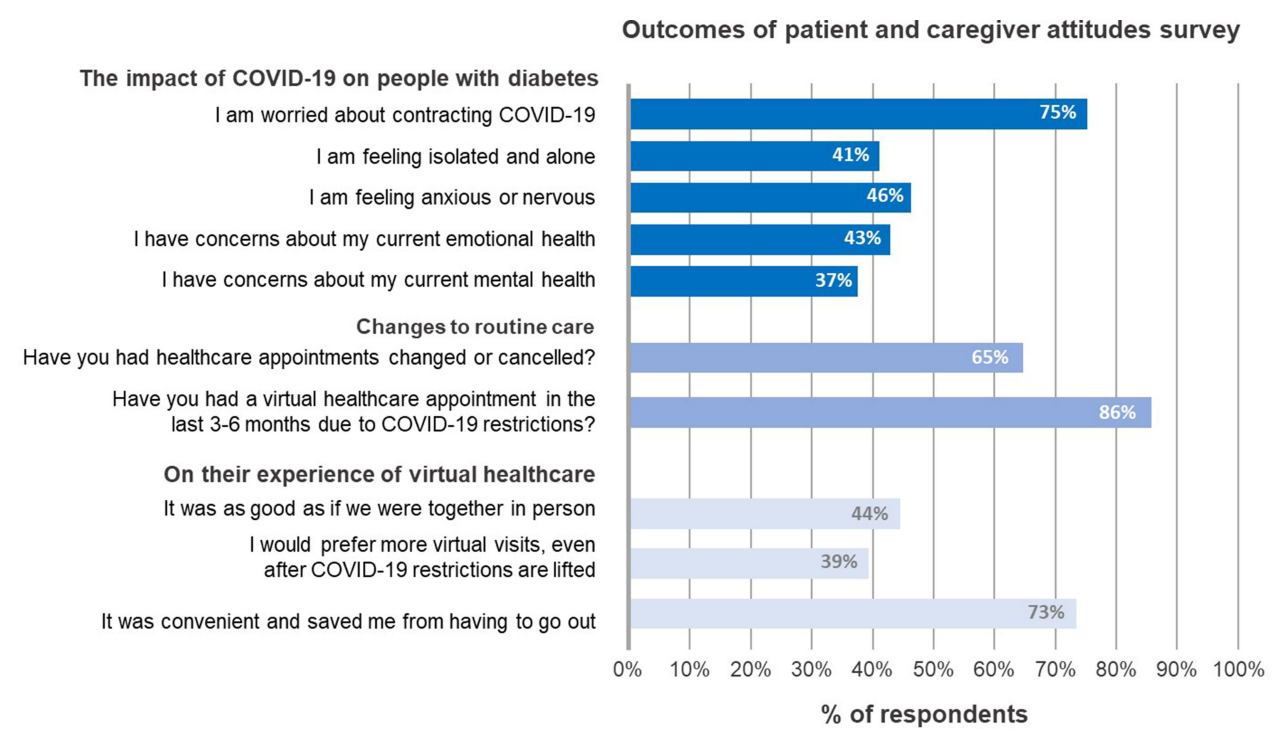

Figure I Key outcomes from people with diabetes and caregivers as a consequence of COVID-I9 pandemic restrictions. Outcomes taken from an online survey conducted by Diabetes Canada. A total of 780 people with diabetes or caregivers provided responses between May I4 and June 17, $2021 .{ }^{44}$

\section{Ipsos Survey of People with Diabetes}

This survey revealed that frustrations with traditional approaches to diabetes self-monitoring (ie, frequent finger pricks) are making PWD consider switching to technology that makes it easier to track their glucose levels digitally between doctors' visits. Key barriers to the use of flash glucose monitoring systems relate primarily to lack of information, but there is a high level of interest in learning more about this option.

Survey respondents were increasingly interested in or actively embracing innovative technology, partly in response to the COVID-19 pandemic and the immediate shift to virtual care. Seventy-three percent of respondents were open to using technology to manage their diabetes, although only 53\% were comfortable using technology to share their health information. Those with T1DM and those who live in Ontario and the Atlantic provinces are generally more open to using technology when it comes to their health.

The pandemic has clearly been a driver of the switch to virtual/digital care, requiring many Canadians to adapt, including those with diabetes. The survey revealed an increasingly receptive attitude towards using technology to manage their diabetes and virtual appointments. Forty-two percent of respondents indicated that they have changed their habits as a result of COVID, including starting to use virtual care or digital resources, using a delivery service for prescriptions, or sharing data with their doctor digitally. One-third of Ontarians have used virtual care and/or other digital resources since the start of the pandemic and $15 \%$ of Ontarians are digitally sharing their glucose data with their doctor.

COVID-19 is also associated with a number of other impacts that are important to address (eg, disruptions to scheduled care, pandemic-related stress about reduced focus on personal diabetes care), both as part of the post-COVID pandemic period and the general move towards technologies in the management of PWD. Respondents also raised questions about whether virtual care is as effective as established care, despite acknowledging that it provides quicker access to HCPs.

\section{Banty Survey of HCPs}

Abbott conducted a survey of HCPs ( $89 \%$ primary care practitioners) centered on their attitudes towards using virtual methods to manage PWD, with a particular focus on use of the FreeStyle Libre system. Overall, the pandemic has been highly disruptive, with significant impacts on regular patient follow-up and laboratory monitoring. The majority of clinicians believe that this has increased health risks for PWD, with 51\% reporting that their patients have experienced an increase in acute episodes leading to a hospital visit.

HCPs have been using a range of tools to maintain contact with their patients, leveraging phone calls, video chats, emails, and/or texts to keep the lines of communication open. The key concerns that HCPs report in dealing with PWD in 
the virtual environment relate to questioning the reliability of the self-reported information; glucose not in the target range; levels of patient stress and anxiety; and, missing laboratory results. Respondents recognize that virtual tools and/or technologies can be helpful for patient education and disease management decision-making and that the pandemic is highlighting the unmet need for new ways to support patient self-management for successful outcomes.

In terms of the FreeStyle Libre system, $71 \%$ of the respondents were confident that it better enables their patients to make more-informed decisions about their disease control, with the same proportion indicating that the system increases patient confidence and can reduce anxiety. The contribution of the FreeStyle Libre system to educating patients and increasing their level of awareness of how various factors directly affect their glucose levels - thus enabling better selfmanagement - was also noted by almost two-thirds of respondents. More than $70 \%$ of the physicians indicated that the FreeStyle Libre system increased their own confidence in making more-informed treatment changes, presumably because of the increased reliability of the data collected.

Notably, $51 \%$ of HCPs noted seeing a reduction in hypoglycemic events and $56 \%$ believe that patients experience better control of their HbAlc levels when using the FreeStyle Libre system. The majority of HCPs indicated that using flash glucose monitoring could contribute to decreasing the risk of diabetes-related comorbidities, complications, hospitalizations, and related health care costs. Almost $60 \%$ of the respondents perceive that the FreeStyle Libre system supports virtual patient care and contributes to continuity of care.

The outcomes from these three surveys indicate that PWD and HCPs have been challenged by COVID-19 and have an appetite for technologies such as the FreeStyle Libre system and the opportunities they provide for a new approach to diabetes management. Virtual care has become established in ensuring continuity of patient care while maintaining pandemic restrictions. Effective sharing of glucose data between patient and HCP has been managed through technologies such as the FreeStyle Libre system. The question now is what ongoing support is needed to leverage digital tools to optimize the care of PWD.

\section{Articulating the Wider Value of the FreeStyle Libre System}

Over and above the clinical trials and real-world data, there is a larger body of research reflecting real-world use and outcomes associated with the FreeStyle Libre system. These data validate the extent of the benefits that the system has brought for PWD.

\section{Benefits for Children and Youth}

The management of T1DM diabetes in children and youth is complex and challenging, so access to tools that can facilitate management and improve outcomes in this population is important. ${ }^{45}$ In addition to the SELFY study noted above, ${ }^{11}$ there have been several studies reporting the benefits of flash glucose monitoring compared to SMBG, including health-related QOL, even if HbA1c has not changed. ${ }^{46-50}$

High levels of satisfaction with the FreeStyle Libre system have been reported by young people with T1DM, ${ }^{46}$ with respondents citing less pain, ease of wearing, discrete scanning, and no impact on daily activities as definable benefits. Improved detection of hypoglycemia is associated with switching from SMBG to the FreeStyle Libre system, with subsequent improved adherence to glucose monitoring. ${ }^{47}$

Flash glucose monitoring enables children to participate in active environments such as summer camps. ${ }^{48,49}$ These studies have demonstrated that flash glucose monitoring is as safe as SMBG in this environment, with improvements in $\mathrm{TIR}^{48}$ and reported high levels of usability and user acceptance.

Retrospective observational analysis of wider access to the FreeStyle Libre system in young PWD is available. A study from Belgium evaluated the use of the FreeStyle Libre system as well as diabetes outcomes in T1DM children and adolescents 1 year after reimbursement. ${ }^{50}$ The system was well accepted overall and resulted in a $53 \%$ decrease in the rate of severe hypoglycemia $(\mathrm{p}=0.012)$ for users, while that rate was unchanged in SMBG users. A multi-centre study carried out in Israel ${ }^{51}$ looking at outcomes over a 6-12-month period showed the shift to the FreeStyle Libre system was associated with significant decreases in mean $\mathrm{HbAlc}$ (from $8.86 \pm 0.23$ to $8.05 \pm 0.2 \% ; \mathrm{p}=0.0001$ ) within the first 3 months of use. 
User acceptance is a particularly important factor in the successful use of the FreeStyle Libre system in children and youth with T1DM. A French study that surveyed 347 children and/or their parents regarding flash glucose monitoring ${ }^{52}$ found that, while the key drivers for using the FreeStyle Libre system were avoidance of painful finger-pricks and the opportunity to check nocturnal glucose levels, there were challenges identified with the use of the system, emphasizing the importance of training and education in this group. This need for education and support to ensure successful implementation is a common theme in pediatric diabetes studies. A prospective study in the UK evaluated the impact of a structured educational approach by HCPs on metabolic outcomes and QOL in children with T1DM. ${ }^{53}$ After 12 months of follow-up, PedsQL3.2 diabetes scores demonstrated significant improvement in patient QOL, and reduction of diabetes symptoms and treatment barriers following initiation with the FreeStyle Libre system.

\section{Benefits for Adults with TIDM or T2DM}

The Flash Monitor Register in the Netherlands (FLARE-NL) study ${ }^{54}$ followed people with T1DM or T2DM using the FreeStyle Libre system for at least 1 year and measured a series of glycemic metrics, changes in well-being and HRQOL, the frequency of hospitalizations related to diabetes, as well as cost-effectiveness and cost-benefit assessments. The FLARE-NL4 study ${ }^{55}$ reported that people using flash glucose monitoring had improved well-being, decreased disease burden, and improved glycemic control after one year of use. Workplace absenteeism and annual diabetes-related hospital admission rates decreased significantly, from $18.5 \%$ to $7.7 \%$ and $13.7 \%$ to $4.7 \%$, respectively. Diabetes-related HRQOL improved, including related mental health. Overall, there was an average decline in HbA1c of $0.4 \%$ associated with the use of the FreeStyle Libre system, and those with higher HbA1c at baseline were likely to see greater reductions with FreeStyle Libre system.

\section{The Burden of Acute Diabetes Events, Hospital Admissions, and Healthcare Economics}

The clinical trials and real-world studies identified earlier attest to the impact of the FreeStyle Libre system in adults with T1DM or T2DM, with a clear focus on reductions in hypoglycemia and HbAlc, among other clinically important measures of glycemic control. The wider impact on the healthcare economy is also becoming clearer.

A logical extension of the evidence that elevated $\mathrm{HbA1c}$ levels are associated with long-term diabetes complications is that they are also associated with increased health system resource utilization. A 2017 real-world study from Ontario ${ }^{56}$ showed that higher HbAlc levels were associated with increased emergency department visits and hospital admissions. We know that the rate of acute diabetes events (ADEs) associated with poorly managed disease is a significant burden on healthcare systems. ADEs such as diabetic ketoacidosis (DKA), hyperglycemia hyperosmolar syndrome (HHS), and severe hypoglycemia (SH) are associated with significant morbidity, mortality, and are a frequent cause of attendance at emergency rooms and hospital admission. These all incur significant and measurable costs. ${ }^{57,58}$

As noted above, the FLARE-NL4 study cohort reported a reduction in diabetes-related hospital admissions from $13.7 \%$ to $4.7 \%$ after starting the FreeStyle Libre system. ${ }^{55}$ As well as showing an association with reduced HbA1c, the ABCD UK FreeStyle Libre system audit also revealed a significant reduction in paramedic calls and hospital admissions due to SH, HHS and DKA. ${ }^{13}$ Given calculated potential costs for each admission of $£ 2152$ for $\mathrm{SH}^{59}$ and $£ 2064$ for DKA $^{58}$ (2014 costs), the use of the FreeStyle Libre system can be linked to significantly reduced health system costs.

The FUTURE study in Belgium ${ }^{60}$ collected real-world data for 1913 adults with T1DM started on the FreeStyle Libre system and showed that hospitalization for SH and/or DKA was 3.3\% in the year prior to introduction of the FreeStyle Libre system and decreased to $2.2 \%$ in the year after $(\mathrm{p}=0.031)$. Another retrospective cohort study in Israel ${ }^{61}$ looked at 3490 adults with T1DM who were initiated on the Freestyle Libre system during 2018 and followed for a median of 14 months. In this group, hospital admissions for DKA were reduced by $48 \%(\mathrm{p}<0.001)$ and for SH admissions were reduced by $43 \%(\mathrm{p}<0.001)$.

The largest study focusing on ADEs in the context of flash glucose monitoring is the RELIEF study, ${ }^{62}$ a retrospective real-world analysis of data from the national French SNDS health claims database, which examined the rate of hospitalizations for ADEs among 74,011 people with T1DM or T2DM following initiation of the Freestyle Libre system, after reimbursement was implemented in 2017. In the first 12 months of Freestyle Libre system use, hospitalizations for 
ADEs fell significantly in T1DM (-49.0\%) and in T2DM (-39.4\%) following initiation of the system. Specifically, admissions for DKA fell in T1DM by $-56.2 \%$ and in T2DM by $-52.1 \%$, with reductions in admissions for diabetesrelated comas in T1DM $(-39.6 \%)$ and in T2DM $(-31.9 \%)$. While the economic implications of these reductions were not assessed, it is fair to assume that such a drop in admissions would lead to health system savings.

In the US, and specifically focusing on the association of the FreeStyle Libre system with ADEs in people with T2DM on rapid-acting insulin therapy, an analysis of healthcare insurance claims databases ${ }^{63}$ found that the rate of ADEs related to hypoglycemia fell by $29 \%$ and that hospital admission rates for any cause decreased by $32 \%$ from $(\mathrm{p}<0.001)$. Of particular note, the reduction in ADEs and hospitalizations was evident within the first 45 days of starting flash glucose monitoring. The potential for these changes to reduce costs was also noted.

Together, these findings at institutional and national levels show that implementing flash glucose monitoring can have significant implications for the acute clinical care of people with T1DM or T2DM, as well as potential economic benefits through reduction in hospital admissions.

\section{The COVID-19 Pandemic: A Case Study in Telemonitoring Using the FreeStyle Libre System}

The use of telemedicine approaches to deliver care for PWD has been evolving for several years ${ }^{64-66}$ and has accelerated as a consequence of the COVID-19 pandemic, given the severely restricted access to in-clinic diabetes services. ${ }^{67}$ As noted above in the patient and HCP surveys, the pandemic has driven a switch to remote monitoring and virtual consultation, both of which can be facilitated by the FreeStyle Libre system. Numerous published studies have demonstrated that glucose control did not deteriorate while access to regular diabetes clinical services was interrupted; and, in many cases, improved across selected metrics ${ }^{68}$ including time in range, time above range, average glucose, and GMI.

The majority of the studies indicated that flash glucose monitoring was associated with successful diabetes management during enforced social distancing. ${ }^{68}$ These include large studies in Scotland ${ }^{69}$ and Spain $^{70}$ demonstrating that $\%$ time in range (\%TIR) 3.9-10 mmol/L $(70-180 \mathrm{mg} / \mathrm{dL})$ over this period increased, with associated improvements in glycemic variability. Smaller studies across Europe involving groups of people with T1DM using the FreeStyle Libre system have typically shown improvements in \%TIR and average glucose during enforced social distancing, ${ }^{71-73}$ including for children and adolescents; ${ }^{74,75}$ and, either improvement or no change in $\%$ time in hypoglycemia over the same period, including for groups at higher risk of hypoglycaemia. ${ }^{76,77}$

Overall, participants who have been able to leverage the FreeStyle Libre system during the COVID-19 pandemic have been successful in managing their disease, particularly when supplemented by virtual interactions with their diabetes team. Given this experience, PWD and HCPs are likely to maintain or increase their use of telemonitoring and telemedicine as part of their approach diabetes care once the pandemic is controlled. ${ }^{78}$

\section{Patient Perspectives and Benefits}

Active engagement and empowerment of PWD in their diabetes care is a key factor in optimizing health outcomes, and diabetes technologies are important tools in achieving these goals. ${ }^{79}$ The Ipsos survey confirmed that there is a high level of interest in technologies that make it easier to digitally track glucose levels between clinic visits. HCPs responding to the Banty Survey noted an important role for technologies in patient education and disease management decisionmaking.

Since PWD and clinicians accept the value of new glucose monitoring systems, it is important to understand how flash glucose monitoring contributes to patient and/or caregiver satisfaction, as well as QOL improvements. Both of these factors are likely to be drivers in successful utilization of this technology and, thus, predictors of the degree of engagement in diabetes care.

\section{Patient Treatment Satisfaction and Well-Being}

The experience and satisfaction of young adults with flash glucose monitoring was evaluated in a cohort with T1DM who were using an insulin pump over a 12 -week period. ${ }^{80}$ Significant improvements in various glucose metrics were evident 
over the study period compared to baseline, including HbAlc, hypoglycemia, and mean capillary glucose. There were also significant improvements in insulin dosing and dietary metrics. Glucose monitoring satisfaction (GMS) was assessed using the T1DM version of the glucose monitoring satisfaction survey (GMSS). There were statistically significant improvements in overall GMS score compared to baseline, as well as improvements in key subdomains: openness, emotional burden, behavioral burden, and trust.

The same researchers assessed diabetes treatment satisfaction (via the DTSQ) and sense of well-being (via the WHO5 Well-Being Index [WHO-5]) with flash glucose monitoring over a 12-week period in a different group of young adults with T1DM. ${ }^{81}$ Compared to baseline, the study group reported significant improvements in treatment satisfaction $(\mathrm{p}<0.001)$ and sense of well-being $(\mathrm{p}<0.001)$ with the system compared with conventional SMBG testing.

A group of Japanese people with T1DM or T2DM being treated with insulin ${ }^{82}$ were evaluated over 14 days and those with T1DM reported significant improvements in both WHO-5 and DTSQ scores using flash glucose monitoring compared to baseline.

\section{Impact on Distress, Fear of Hypoglycemia, and Quality of Life}

A number of studies have focused on changes in psychosocial experiences of people with T1DM using flash glucose monitoring. An assessment of changes in diabetes distress and sleep quality in young adults (mean age 20.9 yrs) with T1DM noted statistically significant improvements in Diabetes Distress Scale (DSS) scores and Pittsburgh Sleep Quality Index metrics over a 3-month period. ${ }^{83}$ Use of flash glucose monitoring was also associated with statistically significant decreases in HbA1c levels and confirmed hypoglycemia episodes per month compared to baseline.

A similar real-world study examined the impact of flash glucose monitoring on glycemic control and diabetes distress in an adult T1DM population in the UK. ${ }^{84}$ As well as significant decreases in HbAlc and a $>50 \%$ reduction in the mean number of hypoglycemic episodes per week, there was also a significant improvement in all five domains of the abbreviated DDS compared to baseline, potentially related to the changes in hypoglycemic episodes.

Fear of hypoglycemia (FOH) is a factor in diabetes distress and lower adherence with therapy. A study ${ }^{85}$ examined changes in FOH among children and young people (13-19 years old) with T1DM after using flash glucose monitoring for 3 months compared to SMBG. Significant improvements were noted in FOH metrics, along with increases in QOL scores, HbAlc, and hypoglycemia in the overall study population. Additionally, these improvements were correlated to more-frequent scanning with the FreeStyle Libre system.

The FUTURE study, ${ }^{60}$ a 12 -month prospective observational study in Belgium, investigated the impact of the FreeStyle Libre system in the year following reimbursement on various measures of QOL and glycemic control in adults with T1DM. Overall, general QOL (SF-36) and diabetes-specific QOL measures (Problem Areas in Diabetes, short form [PAID-SF], Hypoglycemia Fear Survey [HFS]-Worry) were high at baseline and remained stable through the study period, while treatment satisfaction (per the DTSQ) improved significantly and there was a decrease in work absenteeism. These observations align with improvements in treatment satisfaction reported in the IMPACT ${ }^{9}$ and REPLACE RCTs,${ }^{10}$ and with the positive changes in QOL seen in the FLARE-NL-4 study. ${ }^{55}$

Along with the reductions in $\mathrm{HbAlc}$ and reduced ADEs previously noted, the ABCD UK audit ${ }^{14}$ of FreeStyle Libre system users also confirmed that there is a QOL improvement for users, which appears to be related to improved awareness of hypoglycemia and the opportunity to improve overall glycemic control. Improvements in self-care and broader self-esteem were also associated with use of the system.

\section{Limitations and Strengths}

The clinical trials included and real-world evidence reviewed contained only a small number of studies conducted on Canadian subjects and systems, and none on pediatric patients in Canada. The majority of papers covered a variety of non-Canadian populations, and we have made an assumption as to their generalizability to the care of PWD in Canada using sensor-based technology such as the FreeStyle Libre system. Also, the search strategy as outlined did not include the PubMed or SCOPUS databases, as that was deemed to be redundant, given the coverage of the other databases. We therefore cannot fully exclude the possibility of research that counters our conclusions. The original research discussed herein was conducted under a variety of settings, with differing levels of control for confounding factors that may have 
contributed to the treatment effects of sensor-based technologies. We have not ranked studies based on the strength of their outcomes and bias across studies was not assessed.

Strengths of our analysis are the large number of studies identified and the number of children and adult patients included. The papers incorporated also encompass clinical outcomes and healthcare-related quality of life outcomes using validated tools.

\section{Conclusions}

Self-monitoring of glucose is essential in the management of diabetes and sensor-based technologies such as the FreeStyle Libre system are addressing the limitations associated with traditional SMBG. Clinical trials and real-world studies have documented the contribution of the system to improving glucose metrics in people with T1DM and T2DM, particularly as it relates to avoiding hypoglycemic events whilst maintaining or reducing HbAlc levels. A wide range of government agencies and healthcare stakeholders within Canada have confirmed the value and role of the FreeStyle Libre system.

Flash glucose monitoring and CGM systems have helped define a number of new and more dynamic measures of glycemic control, allowing PWD and HCPs to move beyond HbAlc as the sole measure of glucose control. Evidence from real-world practice is confirming that flash glucose monitoring can help patients achieve targets for TIR and reduce time spent in hypoglycemia or hyperglycemia. The correlation between TIR, HbAlc, and diabetes-related morbidity and/ or mortality provides compelling evidence of the value of the FreeStyle Libre system that should be considered by payers. This is clearly highlighted by the strong association between using the FreeStyle Libre system and reduced rates of ADEs and hospitalizations, with predicted consequent reductions in health system costs.

The COVID-19 pandemic has driven a shift towards telemonitoring and provision of diabetes care at a distance. The FreeStyle Libre system has been demonstrated to be a valuable asset for adults and children with diabetes, helping them to maintain or improve successful management of their disease, despite restricted access to regular care.

This current review identifies the evidence-based improvement in a number of glycemic outcomes for PWD in studies assessing the impact of the FreeStyle Libre system up to 12 months. Our future research objectives will encompass the longer-term impact of sustained use of the system beyond 12 months and in different groups of PWD, including the elderly and at-risk, as well as those on basal insulin alone, or on non-insulin therapy. It will also be important to understand the behavioural and lifestyle benefits for PWD using the FreeStyle Libre system.

\section{Medical Writing and Editing}

Editorial assistance in the preparation of this article was provided by Dr Rob Brines of Bite Medical Consulting. Support for this assistance was funded by Abbott Diabetes Care.

\section{Data Sharing Statement}

The data that support our findings are a mix of freely available literature (open access) and subscription publication or restricted-access survey data. The substantial portion of these data are reported here but may not be publicly available.

\section{Ethical Approval}

This article is based on previously published studies or on surveys compliant with respondents' privacy.

\section{Author Contributions}

All named authors contributed to data analysis, drafting or revising the article, have agreed on the journal to which the article will be submitted, gave final approval of the version to be published, and agree to be accountable for all aspects of the work.

\section{Funding}

Sponsorship for this study was provided by Abbott Diabetes Care. 


\section{Disclosure}

JLG has served as a consultant to Abbott Diabetes Care, Canada. LB has served as an advisor/consultant/speaker for Abbott Diabetes Care, Canada and Dexcom Canada. FLG is employee of Abbott Diabetes Care. The authors report no other conflicts of interest in this work.

\section{References}

1. Meetoo D, Wong L, Fatani T. "Knowing where I am": self-monitoring of blood glucose in diabetes. Br J Nurs. 2018;27:537-541. doi:10.12968/ bjon.2018.27.10.537

2. Boutati EI, Raptis SA. Self-monitoring of blood glucose as part of the integral care of type 2 diabetes. Diabetes Care. 2009;32:S205-10. doi: $10.2337 / \mathrm{dc} 09-\mathrm{S} 312$

3. National institute for Health and Care Excellence (NICE). Type 1 diabetes in adults: diagnosis and management: NG17 [Internet]; 2026. Available from: www.nice.org.uk/guidance/ng17. Accessed January 19, 2022.

4. Olansky L, Kennedy L. Finger-stick glucose monitoring. Diabetes Care. 2010;33:948-949. doi:10.2337/dc10-0077

5. Hortensius J, Kleefstra N, Landman GWD, et al. Effects of three frequencies of self-monitored blood glucose on HbAlc and quality of life in patients with type 2 diabetes with once daily insulin and stable control: a randomized trial. BMC Res Notes. 2018;11:26. doi:10.1186/s13104-018-3138-7

6. Polonsky WH, Fisher L, Schikman CH, et al. A structured self-monitoring of blood glucose approach in Type 2 diabetes encourages more frequent, intensive, and effective physician interventions: Results from the STeP Study. Diabetes Technol Ther. 2011;13:797-802. doi:10.1089/dia.2011.0073

7. Association AD. Economic costs of diabetes in the U.S. in 2017. Diabetes Care. 2018;41:917-928. doi:10.2337/dci18-0007

8. Coons MJ, Greiver M, Aliarzadeh B, et al. Is glycemia control in Canadians with diabetes individualized? A cross-sectional observational study. BMJ Open Diabetes Res Care. 2017;5:e000316. doi:10.1136/bmjdrc-2016-000316

9. Bolinder J, Antuna R, Geelhoed-Duijvestijn P, Kröger J, Weitgasser R. Novel glucose-sensing technology and hypoglycaemia in type 1 diabetes: a multicentre, non-masked, randomised controlled trial. Lancet. 2016;388:2254-2263. doi:10.1016/S0140-6736(16)31535-5

10. Haak T, Hanaire H, Ajjan R, Hermanns N, Riveline J-P, Rayman G. Flash glucose-sensing technology as a replacement for blood glucose monitoring for the management of insulin-treated type 2 diabetes: a multicenter, open-label randomized controlled trial. Diabetes Ther. 2017;8:5573. doi:10.1007/s13300-016-0223-6

11. Campbell FM, Murphy NP, Stewart C, Biester T, Kordonouri O. Outcomes of using flash glucose monitoring technology by children and young people with type 1 diabetes in a single arm study. Pediatr Diabetes. 2018;19:1294-1301. doi:10.1111/pedi.12735

12. Yaron M, Roitman E, Aharon-Hananel G, et al. Effect of flash glucose monitoring technology on glycemic control and treatment satisfaction in patients with Type 2 diabetes. Diabetes Care. 2019;42:1178-1184. doi:10.2337/dc18-0166

13. Evans M, Welsh Z, Ells S, Seibold A. The impact of flash glucose monitoring on glycaemic control as measured by HbA1c: a meta-analysis of clinical trials and real-world observational studies. Diabetes Ther Res Treat Educ Diabetes Relat Disord. 2019;11:83-95.

14. Deshmukh H, Wilmot EG, Gregory R, et al. Effect of flash glucose monitoring on glycemic control, hypoglycemia, diabetes-related distress, and resource utilization in the Association of British Clinical Diabetologists (ABCD) nationwide audit. Diabetes Care. 2020;43:2153-2160. doi: $10.2337 / \mathrm{dc} 20-0738$

15. Kröger J, Fasching P, Hanaire H. Three European retrospective real-world chart review studies to determine the effectiveness of flash glucose monitoring on HbAlc in adults with Type 2 diabetes. Diabetes Ther. 2020;11:279-291. doi:10.1007/s13300-019-00741-9

16. Elliott T, Beca S, Beharry R, Tsoukas MA, Zarruk A, Abitbol A. The impact of flash glucose monitoring on glycated hemoglobin in type 2 diabetes managed with basal insulin in Canada: a retrospective real-world chart review study. Diabetes Vasc Dis Res. 2021;18:147916412110213. doi: $10.1177 / 14791641211021374$

17. Wada E, Onoue T, Kobayashi T, et al. Flash glucose monitoring helps achieve better glycemic control than conventional self-monitoring of blood glucose in non-insulin-treated type 2 diabetes: a randomized controlled trial. BMJ Open Diabetes Res Care. 2020;8:e01115. doi:10.1136/bmjdrc2019-001115

18. Wright EE, Kerr MSD, Reyes IJ, Nabutovsky Y, Miller E. Use of flash continuous glucose monitoring is associated with A1C reduction in people with type 2 diabetes treated with basal insulin or noninsulin therapy. Diabetes Spectr. 2021;34:184-189. doi:10.2337/ds20-0069

19. International Hypoglycemia Study Group. Minimizing Hypoglycemia in Diabetes. Diabetes Care. 2015;38:1583-1591. doi:10.2337/dc15-0279

20. Agiostratidou G, Anhalt H, Ball D, Blonde L, Gourgari E, Harriman KN, et al. Standardizing Clinically Meaningful Outcome Measures Beyond HbAlc for Type 1 Diabetes: A Consensus Report of the American Association of Clinical Endocrinologists, the American Association of Diabetes Educators, the American Diabetes Association, the Endocrine Society, JDRF International, The Leona M. and Harry B. Helmsley Charitable Trust, the Pediatric Endocrine Society, and the T1D Exchange. Diabetes Care. 2017; 40:1622-1630.

21. Battelino T, Danne T, Bergenstal RM, et al. Clinical targets for continuous glucose monitoring data interpretation: recommendations from the international consensus on time in range. Diabetes Care. 2019;42(8):1593-1603. doi:10.2337/dci19-0028

22. Beck RW, Bergenstal RM, Cheng P, et al. The relationships between time in range, hyperglycemia metrics, and HbA1c. J Diabetes Sci Technol. 2019;13:614-626. doi:10.1177/1932296818822496

23. Vigersky RA, McMahon C. The relationship of hemoglobin A1C to time-in-range in patients with diabetes. Diabetes Technol The. 2018;21:81-85. doi:10.1089/dia.2018.0310

24. Beck RW, Bergenstal RM, Riddlesworth TD, et al. Validation of time in range as an outcome measure for diabetes clinical trials. Diabetes Care. 2018;42:400-405. doi:10.2337/dc18-1444

25. Lu J, Ma X, Zhou J, et al. Association of time in range, as assessed by continuous glucose monitoring, with diabetic retinopathy in type 2 diabetes. Diabetes Care. 2018;41(11):2370-2376. doi:10.2337/dc18-1131

26. Li F, Zhang Y, Li H, et al. TIR generated by continuous glucose monitoring is associated with peripheral nerve function in type 2 diabetes. Diabetes Res Clin Pr. 2020;166:108289. doi:10.1016/j.diabres.2020.108289

27. Lu J, Ma X, Shen Y, et al. Time in range is associated with carotid intima-media thickness in type 2 diabetes. Diabetes Technol The. 2020;22:72-78. doi:10.1089/dia.2019.0251 
28. Lu J, Wang C, Shen Y, et al. Time in range in relation to all-cause and cardiovascular mortality in patients with type 2 diabetes: a prospective cohort study. Diabetes Care. 2021;44:549-555. doi:10.2337/dc20-1862

29. Monnier L, Colette C, Wojtusciszyn A, et al. Toward defining the threshold between low and high glucose variability in diabetes. Diabetes Care. 2017;40(7):832-838. doi:10.2337/dc16-1769

30. Bergenstal RM, Beck RW, Close KL, et al. Glucose Management Indicator (GMI): a new term for estimating a1c from continuous glucose monitoring. Diabetes Care. 2018;41(11):2275-2280. doi:10.2337/dc18-1581

31. Bergenstal RM, Ahmann AJ, Bailey T, et al. Recommendations for standardizing glucose reporting and analysis to optimize clinical decision making in diabetes: the ambulatory glucose profile. J Diabetes Sci Technol. 2013;7(2):562-578. doi:10.1177/193229681300700234

32. Ceriello A, Monnier L, Owens D. Glycaemic variability in diabetes: clinical and therapeutic implications. Lancet Diabetes Endocrinol. 2018;7 (3):221-230. doi:10.1016/S2213-8587(18)30136-0

33. Palylyk-Colwell E, Ford C. Flash glucose monitoring for diabetes. Can Agency Drugs Technol Health. 2016;158:1-13.

34. Boucher MC. Flash glucose monitoring system - FreeStyle Libre to monitor glycemia in patients with diabetes. Report No: 2369-7385; 2020.

35. CADTH. Implementation advice: freeStyle Libre flash glucose monitoring system. Report; 2020.

36. Young C, Grobelna A. Flash glucose monitoring systems in pediatric populations with diabetes. Can J Health Technol. 2021;1:1-67. doi:10.51731/ cjht. 2021.60

37. OntarioHealth:quality. Ontario health technology advisory committee [Internet]. Available from: https://hqontario.ca/Evidence-to-Improve-Care/ Health-Technology-Assessment/Ontario-Health-Technology-Advisory-Committee. Accessed January 19, 2022.

38. OntarioHealth:Quality. Ontario health technology advisory committee terms of reference [Internet]. Available from: https://hqontario.ca/portals/0/ documents/evidence/reports/ohtac-terms-of-reference-en.pdf. Accessed January 19, 2022.

39. OntarioHealth:Quality. Flash glucose monitoring system for people with type 1 or type 2 diabetes: a health technology assessment; 2019.

40. INESSS. Evaluation process and criteria [Internet]. Available from: https://www.inesss.qc.ca/en/themes/medicaments/evaluation-process-and-cri teria.html. Accessed January 19, 2022.

41. INESSS. Avis - Système flashed surveillance du glucose. [Guide - Flash Glucose Monitoring System]. (FreeStyle Libre, Abbott); 2018.

42. FreeStyle Libre - Diabète. Avis transmis à la ministre en Mars; [FreeStyle Libre - Diabetes. Advice from the Minister]; 2020. [Press release].

43. Cheng AYY, Feig DS, Ho J, et al.; Diabetes Canada Clinical Practice Guidelines Expert Working Group. Blood glucose monitoring in adults and children with diabetes: update 2021. Can J Diabetes. 45;2021:580-587. doi:10.1016/j.jcjd.2021.07.003

44. Diabetes Canada. Results of patient \& caregiver survey; 2021. Available from: https://www.diabetes.ca/DiabetesCanadaWebsite/media/Campaigns/ COVID-19\%20and\%20Diabetes/2021-Patient-Caregiver-Survey-Results.pdf. Accessed January 19, 2022.

45. Wherrett DK, Ho J, Huot C, et al.; Diabetes Canada Clinical Practice Guidelines Expert Working Group. Type 1 diabetes in children and adolescents. Can J Diabetes. 42;2018:S234-46. doi:10.1016/j.jcjd.2017.10.036

46. Massa GG, Gys I, Eyndt AO, et al. Evaluation of the FreeStyle ${ }^{\circledR}$ Libre flash glucose monitoring system in children and adolescents with type 1 diabetes. Horm Res Paediat. 2018;89:189-199. doi:10.1159/000487361

47. Deeb A, Yousef H, Qahtani NA, et al. Novel ambulatory glucose-sensing technology improves hypoglycemia detection and patient monitoring adherence in children and adolescents with type 1 diabetes. J Diabetes Metabolic Disord. 2019;18:1-6. doi:10.1007/s40200-018-0351-9

48. Piona C, Dovc K, Mutlu GY, et al. Non-adjunctive flash glucose monitoring system use during summer-camp in children with type 1 diabetes: the free-summer study. Pediatr Diabetes. 2018;19:1285-1293. doi:10.1111/pedi.12729

49. Deja G, Kłeczek M, Chumięcki M, et al. The usefulness of the FlashStyle Libre system in glycemic control in children with type 1 diabetes during summer camp. Pediatric Endocrinol Diabetes Metabolism. 2018;24:11-19. doi:10.18544/PEDM-24.01.0098

50. Messaaoui A, Tenoutasse S, Crenier L. Flash glucose monitoring accepted in daily life of children and adolescents with type 1 diabetes and reduction of severe hypoglycemia in real-life use. Diabetes Technol Ther. 2019;21:329-335. doi:10.1089/dia.2018.0339

51. Landau Z, Abiri S, Gruber N, et al. Use of flash glucose-sensing technology (FreeStyle Libre) in youth with type 1 diabetes: aWeSoMe study group real-life observational experience. Acta Diabetol. 2018;55:1303-1310. doi:10.1007/s00592-018-1218-8

52. Vergier J, Samper M, Dalla-Vale F, et al. Evaluation of flash glucose monitoring after long-term use: a pediatric survey. Prim Care Diabetes. 2019;13:63-70. doi:10.1016/j.pcd.2018.08.004

53. Pintus D, Ng SM. Freestyle libre flash glucose monitoring improves patient quality of life measures in children with Type 1 diabetes mellitus (T1DM) with appropriate provision of education and support by healthcare professionals. Diabetes Metabolic Syndrome Clin Res Rev. 2019;13:2923-2926. doi:10.1016/j.dsx.2019.07.054

54. Fokkert MJ, Damman A, Dijk PR, et al. Use of freestyle libre flash monitor register in the Netherlands (FLARE-NL1): Patient experiences, satisfaction, and cost analysis. Int J Endocrinol. 2019;2019:1-6. doi:10.1155/2019/4649303

55. Fokkert M, Dijk P, Edens M, et al. Improved well-being and decreased disease burden after 1-year use of flash glucose monitoring (FLARE-NL4). BMJ Open Diabetes Res Care. 2019;7:e000809. doi:10.1136/bmjdrc-2019-00080

56. Birtwhistle R, Green ME, Frymire E, et al. Hospital admission rates and emergency department use in relation to glycated hemoglobin in people with diabetes mellitus: a linkage study using electronic medical record and administrative data in Ontario. CMAJ Open. 2017;5:E557-64. doi:10.9778/cmajo.20170017

57. Desai D, Mehta D, Mathias P, Menon G, Schubart UK. Health care utilization and burden of diabetic ketoacidosis in the U.S. over the past decade: a nationwide analysis. Diabetes Care. 2018;41:1631-1638. doi:10.2337/dc17-1379

58. Dhatariya KK, Skedgel C, Fordham R. The cost of treating diabetic ketoacidosis in the UK: a national survey of hospital resource use. Diabetic Med. 2017;34:1361-1366. doi:10.1111/dme.13427

59. Parekh WA, Ashley D, Chubb B, Gillies H, Evans M. Approach to assessing the economic impact of insulin-related hypoglycaemia using the novel Local Impact of Hypoglycaemia Tool. Diabetic Med. 2015;32:1156-1166. doi:10.1111/dme.12771

60. Charleer S, Block CD, Huffel LV, et al. Quality of life and glucose control after 1 year of nationwide reimbursement of intermittently scanned continuous glucose monitoring in adults living with type 1 diabetes (FUTURE): a Prospective Observational Real-World Cohort Study. Diabetes Care. 2020;43:389-397. doi:10.2337/dc19-1610

61. Tsur A, Cahn A, Israel M, Feldhamer I, Hammerman A, Pollack R. Impact of flash glucose monitoring on glucose control and hospitalization in type 1 diabetes: a nationwide cohort study. Diabetes Metabolism Res Rev. 2020;e3355. doi:10.1002/dmrr.3355 
62. Roussel R, Riveline J-P, Vicaut E, et al. Important drop rate of acute diabetes complications in people with type 1 or type 2 diabetes after initiation of flash glucose monitoring in France: the RELIEF Study. Diabetes Care. 2021;44(6):1368-1376. doi:10.2337/dc20-1690

63. Bergenstal RM, Kerr MSD, Roberts GJ, Souto D, Nabutovsky Y, Hirsch IB. Flash CGM is associated with reduced diabetes events and hospitalizations in insulin-treated type 2 diabetes. $J$ Endocr Soc. 2021;5:bvab013. doi:10.1210/jendso/bvab013

64. Wood CL, Clements SA, McFann K, Slover R, Thomas JF, Wadwa RP. Use of telemedicine to improve adherence to American Diabetes Association standards in pediatric type 1 diabetes. Diabetes Technol The. 2016;18:7-14. doi:10.1089/dia.2015.0123

65. Tonyushkina KN, Cobb V, Moskovitz A, Allen HF. Televisits to partially substitute for clinic visits are feasible and well accepted by tech-savvy patients with T1DM and their families: a Prospective Pilot Study. J Diabetes Sci Technol. 2018;12:1084-1085. doi:10.1177/1932296818775438

66. McGloin H, O'Connell D, Glacken M, et al. Patient empowerment using electronic telemonitoring with telephone support in the transition to insulin therapy in adults with type 2 diabetes: observational, pre-post, mixed methods study. J Med Internet Res. 2020;22(5):e16161. doi:10.2196/ 16161

67. Glazier RH, Green ME, Wu FC, Frymire E, Kopp A, Kiran T. Shifts in office and virtual primary care during the early COVID-19 pandemic in Ontario, Canada. CMAJ. 2021;193:E200-10. doi:10.1503/cmaj.202303

68. Danne T, Limbert C, Domingo MP, et al. Telemonitoring, telemedicine and time in range during the pandemic: paradigm change for diabetes risk management in the post-COVID future. Diabetes Ther. 2021;12:2289-2310. doi:10.1007/s13300-021-01114-x

69. Dover AR, Ritchie SA, McKnight JA, et al. Assessment of the effect of the COVID-19 lockdown on glycaemic control in people with type 1 diabetes using flash glucose monitoring. Diabetic Med. 2020;38(1):e14374. doi:10.1111/dme.14374

70. Fernández E, Cortazar A, Bellido V. Impact of covid-19 lockdown on glycemic control in patients with type 1 diabetes. Diabetes Res Clin Pr. 2020;166:108348. doi:10.1016/j.diabres.2020.108348

71. Bonora BM, Boscari F, Avogaro A, Bruttomesso D, Fadini GP. Glycaemic control among people with type 1 diabetes during lockdown for the SARS-CoV-2 outbreak in Italy. Diabetes Ther. 2020;11:1369-1379. doi:10.1007/s13300-020-00829-7

72. Aragona M, Rodia C, Bertolotto A, et al. Type 1 diabetes and COVID-19: the "Lockdown effect.". Diabetes Res Clin Pr. 2020;170:108468. doi:10.1016/j.diabres.2020.108468

73. Capaldo B, Annuzzi G, Creanza A, et al. Blood glucose control during lockdown for COVID-19: CGM metrics in Italian adults with type 1 diabetes. Diabetes Care. 2020;2:dc201127.

74. Marigliano M, Maffeis C. Glycemic control of children and adolescents with type 1 diabetes improved after COVID-19 lockdown in Italy. Acta Diabetol. 2021;43:1-4.

75. Schiaffini R, Barbetti F, Rapini N, et al. School and pre-school children with type 1 diabetes during Covid-19 quarantine: the synergic effect of parental care and technology. Diabetes Res Clin Pr. 2020;166:108302. doi:10.1016/j.diabres.2020.108302

76. Mesa A, Viñals C, Pueyo I, et al. The impact of strict COVID-19 lockdown in Spain on glycemic profiles in patients with type 1 Diabetes prone to hypoglycemia using standalone continuous glucose monitoring. Diabetes Res Clin Pr. 2020;167:108354. doi:10.1016/j.diabres.2020.108354

77. Maddaloni E, Coraggio L, Pieralice S, Carlone A, Pozzilli P, Buzzetti R. Effects of COVID-19 lockdown on glucose control: continuous glucose monitoring data from people with diabetes on intensive insulin therapy. Diabetes Care. 2020;56:dc200954.

78. Murphy H. Managing diabetes in pregnancy before, during and after COVID-19. Diabetes Technol The. 2020;22:454-461. doi:10.1089/ dia.2020.0223

79. Alcántara-Aragón V. Improving patient self-care using diabetes technologies. Ther Adv Endocrinol Metabolism. 2019;10:2042018818824215. doi:10.1177/2042018818824215

80. Hayek AAA, Robert AA, Dawish MAA. Differences of freestyle libre flash glucose monitoring system and finger pricks on clinical characteristics and glucose monitoring satisfactions in type 1 diabetes using insulin pump. Clin Medicine Insights Endocrinol Diabetes. 2019;12:1179551419861102. doi:10.1177/1179551419861102

81. Hayek AAA, Dawish MAA. The potential impact of the freestyle libre flash glucose monitoring system on mental well-being and treatment satisfaction in patients with type 1 diabetes: a prospective study. Diabetes Ther. 2019;10:1239-1248. doi:10.1007/s13300-019-0616-4

82. Mitsuishi S, Nishimura R, Harashima S, et al. The effect of novel glucose monitoring system (flash glucose monitoring) on mental well-being and treatment satisfaction in Japanese people with diabetes. Adv Ther. 2018;35:72-80. doi:10.1007/s12325-017-0649-x

83. Hayek AAA, Dawish MAA. Assessing diabetes distress and sleep quality in young adults with type 1 diabetes using freestyle libre: a prospective cohort study. Diabetes Ther. 2020;11:1551-1562. doi:10.1007/s13300-020-00849-3

84. Nana M, Moore SL, Ang E, Lee ZX, Bondugulapati LNR. Flash glucose monitoring: impact on markers of glycaemic control and patient-reported outcomes in individuals with type 1 diabetes mellitus in the real-world setting. Diabetes Res Clin Pr. 2019;157:107893. doi:10.1016/j. diabres.2019.107893

85. Hayek AAA, Robert AA, Dawish MAA. Evaluation of freestyle libre flash glucose monitoring system on glycemic control, health-related quality of life, and fear of hypoglycemia in patients with type 1 diabetes. Clin Medicine Insights Endocrinol Diabetes. 2017;10:1179551417746957. doi:10.1177/1179551417746957

86. Ontario Ministry of Health, Drugs and Devices Division. Notice from the executive officer: funding flash glucose monitoring system through the Ontario drug benefit program; 2019.

87. The flash glucose monitoring system is now covered [press release]. Diabetes Quebec; 2019.

88. Yukoners with Type 1 Diabetes receive continued support [press release]. Government of Yukon; 2020.

89. Canada ADC. Public and private coverage for the FreeStyle Libre system - what you need to know. [Internet]. Available from: https://www. freestyle.abbott/ca-en/home/freestyle-libre.html. Accessed January 19, 2022.

90. Province invests in new diabetes supports for young Manitobans [press release]. Available from: https://news.gov.mb.ca/news/index.html?item= 52440. Accessed September 2021.

91. Budget 2021-22 continues to build upon record investments in Saskatchewan health care [press release]. Government of Saskatchewan; 2021.

92. Enhanced supports for Saskatchewan residents with diabetes [press release]. Government of Saskatchewan; 2021. 


\section{Publish your work in this journal}

ClinicoEconomics and Outcomes Research is an international, peer-reviewed open-access journal focusing on Health Technology Assessment, Pharmacoeconomics and Outcomes Research in the areas of diagnosis, medical devices, and clinical, surgical and pharmacological intervention. The economic impact of health policy and health systems organization also constitute important areas of coverage. The manuscript management system is completely online and includes a very quick and fair peer-review system, which is all easy to use. Visit http://www.dovepress.com/testimonials.php to read real quotes from published authors.

Submit your manuscript here: https://www.dovepress.com/clinicoeconomics-and-outcomes-research-journal 